\title{
Non-typical Symptomatic Appendico-Vesical Fistula
}

\section{Woo Seok Kim}

Department of Surgery, Soonchunhyang University Gumi Hospital, Gumi, Korea

\begin{abstract}
Appendico-vesical fistula is rare and occurs secondary to acute or missed acute appendicitis. It was first described in 1898. Since then, over 110 cases have been reported in the literature. We report a case of a 46-year-old male with a non-typical symptomatic appendico-vesical fistula.
\end{abstract}

Keywords: Appendix; Appendico-vesical fistula; Appendicitis

\section{INTRODUCTION}

Appendico-vesical fistula (AVF) is rare and occurs secondary to acute or missed acute appendicitis. Early diagnosis of AVF is difficult because the symptoms are ambiguous. The underlying pathology in most cases is acute suppurative appendicitis [1]. We present a case that spontaneously developed non-typical symptomatic AVF after a history of treatment with antibiotics after suspected acute appendicular colic pain 7 years earlier.

\section{CASE REPORT}

A 46-year-old healthy male presented with a 2-month history of epigastric and right lower abdominal discomfort. He had experienced severe pain in the right lower abdomen 10 years previously and had been treated with antibiotics. Intermittent pneumaturia had occurred up to 4 years before the present admission. Thereafter, he did not visit the hospital because he had been no history of chills, fever, or other urinary symptoms.

On physical examination, mild tenderness was apparent in the right lower abdomen with no rebound tenderness. Laboratory examination of blood and urine showed normal findings except for elevated C-reactive protein $(7.29 \mathrm{mg} / \mathrm{dL})$. Abdominopelvic computed tomography $(\mathrm{CT})$ revealed focal appendicitis at the tip of the appendix with appendicolith and firm adhesion between the ap- pendix and bladder, as well as air in the bladder, suggesting fistula (Fig. 1). With strong suspicion of AVF, surgical exploration without cystoscopy was decided upon.

Laparoscopy was performed through three abdominal ports. The distal tip of the long appendix firmly adhered to the right lateral side of the bladder with thick fibrosis. The body and base of the appendix looked healthy without surrounding fibrosis (Fig. 2). No ascites or abscess was observed. The base of the appendix was ligated with 2-0 Vicryl. After removing the proximal appendix, dissection of the bladder around the tip of the appendix was performed. Partial cystectomy was performed and laparoscopic twolayered bladder repair was done with 3-0 Vicryl. And a urethral catheter was placed. In the specimen, the opening is identified on the bladder side (Fig. 3). The abdominal cavity was closed after placing a drain.

A urethral catheter was removed 7 days after the surgery. The histopathologic diagnosis was chronic appendicitis with benign fibro-musculo-adipose tissue. The postoperative course was uneventful, and the patient was discharged 10 days after the operation. The patient provided written informed consent for the publication of clinical details and images.

\section{DISCUSSION}

Enterovesical fistula is a rare complication of malignancy and
Correspondence to: Woo Seok Kim

Department of Surgery, Soonchunhyang University Gumi Hospital, 179 1(illgongdan-ro, Gumi 39371, Korea

Tel: +82-54-468-9093, Fax: +82-54-468-9097, E-mail: 71530@schmc.ac.kr

Received: Apr. 29, 2020 / Accepted after revision: Jun. 18, 2020
(C) 2020 Soonchunhyang Medical Research Institute This is an Open Access article distributed under the terms of the Creative Commons Attribution Non-Commercial License (https://creativecommons.org/licenses/by-nc/4.0/). 


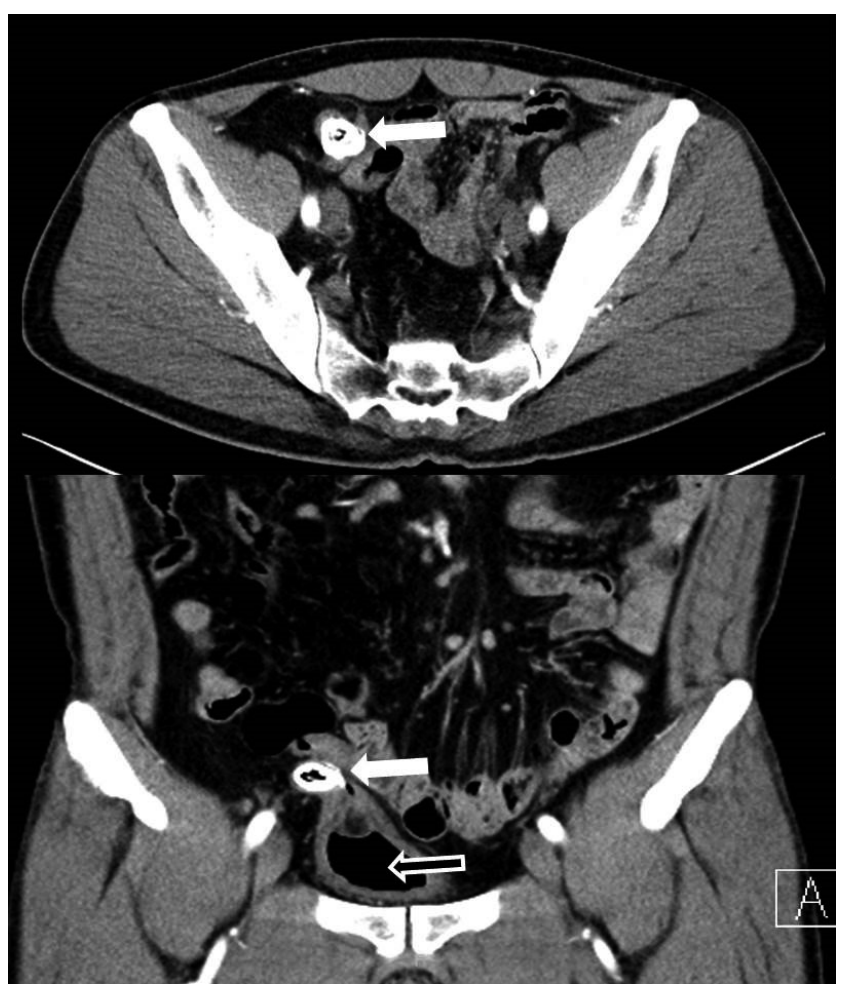

Fig. 1. Computed tomography findings. Focal appendicitis at the tip of the appendix with appendicolith (white arrow) and firm adhesion between the appendix and bladder, as well as air in the bladder (empty arrow) suggesting fistula.

inflammation. The common causes of enterovesical fistula include colonic diverticulitis (51\%), colorectal cancer (16\%), Crohn's disease (12\%), and bladder cancer (5\%) [2]. Additionally, AVFs are extremely rare, accounting for only $1 \%-5 \%$ of all enterovesical fistulas [1]. The underlying pathology in most cases is a failure to recognize and treat acute appendicitis. It was first described in 1898 [3]. Since then over 110 cases have been reported in the literature.

AVFs are often reported in young males 10 to 40 years of age, reflecting the increased incidence of appendicitis in that age group. The higher incidence of this disease in males originates from the uterine interposition between the bladder and appendix in females [4]. Although the cause of fistula is appendicitis, the symptoms of appendicitis are not permanent because of the drainage inside the bladder, but they reappear when the lumen is obstructed. A clinical presentation consistent with appendicitis is uncommon since patients are usually treated by appendicectomy before the fistula develops. AVF is usually evident by the periodic appearance and remission of the following symptoms, ranked in descending order of their frequency: urinary tract infection, frequency, burning,

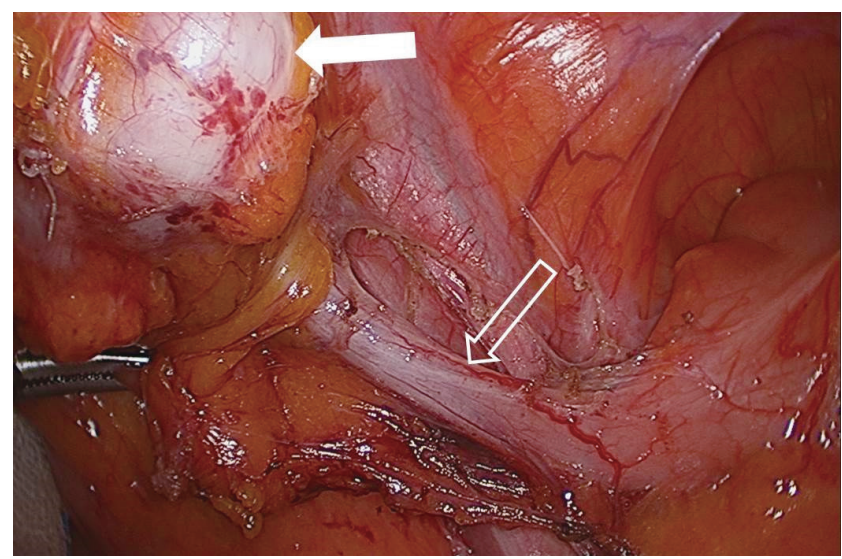

Fig. 2. Laparoscopy showing the distal tip of the long appendix (white arrow) was firmly adhered to the bladder with thick fibrosis. The body and base of the appendix (empty arrow) looked healthy without surrounding fibrosis.

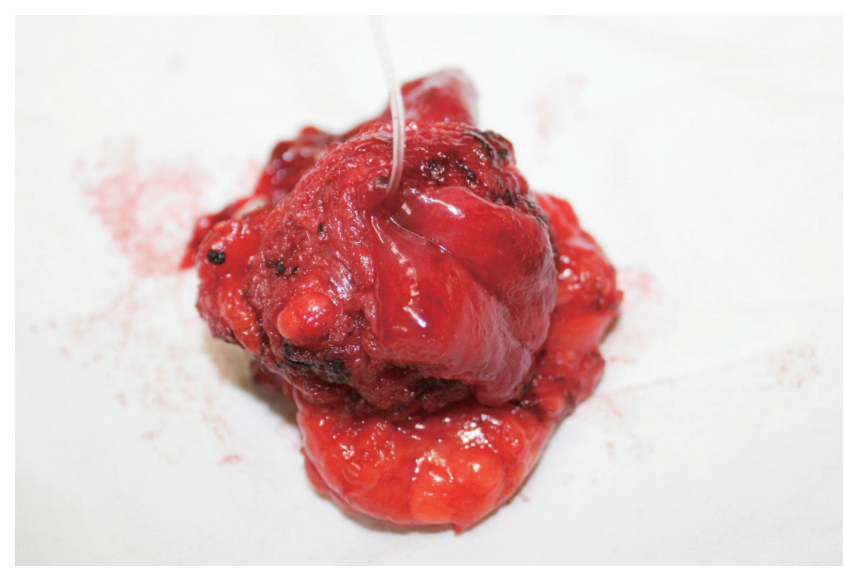

Fig. 3. Specimen. The fistula tract and opening were identified on bladder side.

and dysuria (90\%); abdominal pain (50\%); fever and chills (42\%); hematuria and fecaluria (38\%); diarrhea (35\%); bladder calculi (28\%); the remote history of abdominal pain as an initial symptom and pneumaturia (25\%); anorexia and loss of weight (20\%); nausea and vomiting (18\%); obstruction and retention (15\%); orchitis, epididymitis, and balanitis (13\%); and abdominal or rectal mass (10\%) [1]. Pneumaturia and fecaluria are not always observed because the passage of air or feces through the fistula may be prevented by a fecalith or the long narrow lumen of the appendix.

However, early diagnosis of AVF is difficult because the symptoms are occasionally ambiguous, and useful diagnostic tools have not yet been identified. It usually takes at least 1 year from the onset symptoms to diagnosis [5]. Diagnosis may be delayed for more than 10 years [5]. The diagnostic tools used for the diagnosis of AVF include physical examination, intravenous pyelography, cys- 
toscopy, retrograde cystography, barium enema, colonoscopy, and CT scans. Stones in the bladder (fecaliths) and bullous edema of the bladder wall on cystoscopy raise the possibility of enterovesical fistula. Cystoscopy is sometimes useful in patients showing a fistulous opening in the bladder wall. Cystography is also a useful diagnostic tool in patients who demonstrate a fistula tract between the bladder and appendix. Barium enema examination may demonstrate the contrast in the appendix approaching or entering the bladder. A barium enema is usually negative but the presence of contrast in the fistula's tract or the bladder can be revealed by repeat films taken within 12-24 hours. AVF has not been diagnosed at colonoscopy, but this investigation is important to exclude intestinal pathology. Recently, multislice CT or magnetic resonance imaging with the desired cross-section has been described as being helpful in detecting these fistulas. The presence of intravesical gas on a CT scan indicates the presence of a fistula assuming no prior instrumentation of the urinary tract, but the origin of the fistula may not be demonstrated. Findings consistent with AVF include an abscess cavity or inflammatory mass between the caecum and bladder, and thickening or calcification of the bladder wall [6]. Goldman et al. [7] reported being unable to find all the enterovesical fistula with 1-cm interval CT. Yamamoto et al. [8] described a case where all investigations were negative and the diagnosis was made by laparoscopy. These descriptions indicate that the probability of correct diagnosis is still low. Comparatively, abdominal CT is the most useful tool [9].

Appendectomy or ileocecal resection is required, and partial cystectomy can be added for complete resection of AVF [1]. Laparoscopic procedures should be considered and might be more helpful in appropriate situations. A recent report described an
AVF that was successfully treated with laparoscopic appendectomy and partial cystectomy using an endolinear stapler to resect the bladder cuff involving the fistula [10].

AVF is extremely rare, and it is very difficult to diagnose preoperatively because the symptoms are vague and diagnostic tools cannot readily identify the disease. When we suspect appendicitis with an atypical history, we should carefully examine patients with multiple modalities and the risk of such severe and rare complication of AVF should not be ignored.

\section{REFERENCES}

1. Haas GP, Shumaker BP, Haas PA. Appendicovesical fistula. Urology 1984; 24:604-9.

2. Carson CC, Malek RS, Remine WH. Urologic aspects of vesicoenteric fistulas. J Urol 1978;119:744-6.

3. Keen WW. A case of appendicitis in which the appendix became permanently soldered to bladder, like a third ureter, producing a urinary fecal fistula. Trans Am Surg Assoc 1898;16:243.

4. Abubakar AM, Pindiga UH, Chinda JY, Nggada HA. Appendicovesical fistula associated with Hirschsprung's disease. Pediatr Surg Int 2006;22: 617-8.

5. Bigler ME, Wofford JE, Pratt SM, Stone WJ. Serendipitous diagnosis of appendicovesical fistula by bone scan: a case report. J Urol 1989;142:815-6.

6. Fraley EE, Reinberg Y, Holt T, Sneiders A. Computerized tomography in the diagnosis of appendicovesical fistula. J Urol 1993;149:830-2.

7. Goldman SM, Fishman EK, Gatewood OM, Jones B, Siegelman SS. CT in the diagnosis of enterovesical fistulae. AJR Am J Roentgenol 1985;144: 1229-33.

8. Yamamoto H, Yoshida M, Sera Y, Ikeda S, Terakura H. Laparoscopic diagnosis of appendicovesical fistula in a pediatric patient. Surg Laparosc Endosc 1997;7:266-7.

9. Jarrett TW, Vaughan ED Jr. Accuracy of computerized tomography in the diagnosis of colovesical fistula secondary to diverticular disease. J Urol 1995;153:44-6.

10. Chung CW, Kim KA, Chung JS, Park DS, Hong JY, Hong YK. Laparoscopic treatment of appendicovesical fistula. Yonsei Med J 2010;51:463-5. 